\title{
Liability in the context of misdiagnosis of melanoma in Australia
}

\section{Melody R Abikhair MB BS(Hons) Medical Officer \\ Patrick D Mahar MB BS(Hons), LLB(Hons), MBA Clinical Fellow, ${ }^{2}$ and Dermatology Registrar ${ }^{3}$ \\ Adrian R Cachia FRCPA, LLB(Hons), FACLM, \\ Dermatopathologist \\ John W Kelly \\ MB BS, MD, FACD Director, ${ }^{5}$ and Clinical Associate Professor of Dermatology ${ }^{6}$ \\ 1Department of Dermatology, Monash Medical Centre, Monash Health \\ Melbourne, VIC \\ 2 Department of Medicine St Vincent's Hospital, University of Melbourne, \\ Melbourne, VIC. \\ 3 Department of Dermatology, St Vincent's Hospital, \\ St Vincent's Health, \\ Melbourne, VIC \\ 4 Skin and Cancer Foundation Australia Sydney, NSW. \\ 5 Victorian Melanoma Service, The Alfred Hospital, Alfred Health, \\ Melbourne, VIC \\ 6 Faculty of Medicine Nursing and Health Sciences, Monash University, Melbourne, VIC. \\ pmahar@ \\ student.unimelb.edu.au}

doi: 10.5694/mjal3.10239
$\mathrm{N}$ alignant melanoma is a disease for which misdiagnosis may have very serious ramifications for both patients and clinicians. Given how uncertain and difficult the diagnosis of some melanomas can be, clinicians may well be apprehensive about their potential professional liability arising from claimed misdiagnosis or mismanagement of melanoma. A recent Supreme Court of New South Wales decision ${ }^{1}$ is one of few Australian cases to directly address this issue specifically in relation to melanoma. Coote $v$ Dr Kelly exists in the context of recent High Court of Australia decisions relating to the common law of professional negligence in Australia.

Therefore, it is important to examine the particular facts of the case, how it was decided and why, and whether the court's decision can reasonably be reconciled with what is understood of melanoma diagnosis clinically and from evidence-based medicine as well as a subsequent appeal which resulted in an order for retrial. We emphasise the importance of early recognition of uncertainty in diagnosis and subsequent escalation, particularly where delayed diagnosis may affect survival. This article provides medical practitioners with a better understanding of the

uncertainty inherent in the law regarding certain issues of causation in negligence cases, and gives some guidance on an appropriate standard of care in the diagnosis of melanoma.

\section{Coote v Dr Kelly: the facts}

In September 2009, a patient consulted his general practitioner about a lesion on the plantar surface of his foot, which was diagnosed and subsequently treated as a plantar wart. Despite repeated attempts at cryotherapy, paring and topical treatments to the lesion, it continued to enlarge and change in shape and colour over the following 18 months. During this time, the patient was seen by the same doctor and, subsequently, by two other clinicians, all of whom continued to treat the lesion as a plantar wart. By March 2011, the lesion was noted to have substantially increased in size and to have ulcerated. It was then excised and diagnosed histologically as an invasive acral lentiginous melanoma (ALM). The lesion had metastasised, and the plaintiff faced a poor prognosis. Proceedings in negligence against the initial treating GP were brought before the NSW Supreme Court. Despite some uncertainty about the initial appearance of the lesion, the court found that the GP had breached his duty of care by failing to perform a biopsy on the lesion at an earlier stage; had he done so, it may have led to an earlier diagnosis of ALM. However, all the elements for

Malignant melanoma is a serious and relatively common
condition, the diagnosis of which may be difficult.
misdiagnosis of melanoma occurred, but there was
failure to establish causation of the patient's poor
prognosis.
Aggressive melanomas may grow quickly, fail to conform
to standard and commonly taught diagnostic criteria,
and frequently escape early detection.
In the event of uncertain diagnosis or failed treatment of
a lesion, an appropriate standard of care is full excisional
biopsy if not previously performed, or referral of the case
to an appropriate specialist or melanoma centre.
Clinicians should remain aware of the existence of
higher-risk, easily misdiagnosed melanomas with a high
mortality rate. Therefore, they should aim to identify
these at the earliest opportunity.

negligence were not established. There was insufficient evidence to show that the breach of duty had caused the ultimate harm that befell the patient and, specifically, that an earlier diagnosis of ALM would have prevented the metastasis and subsequent poor prognosis.

\section{Standard of care and breach of duty}

\section{Judicial findings}

The court determined that the appropriate clinical standard of care was not met. A breach of duty of care was established, which was held to constitute the GP's failure to observe a small black mark in the lesion at the initial consultation. The court determined that this ought to have drawn the attention of a reasonably competent practitioner to the need for further investigation. However, the New South Wales Court of Appeal has ordered a retrial (pending) on the basis of both flawed reasoning leading to the original conclusion of breach of duty, and flawed reasoning that such a breach, if it occurred, could nevertheless not be proven on the balance of probabilities to have caused the patient's loss by using evidence of population-aggregated survival statistics. ${ }^{2}$

\section{Commentary}

Certain melanomas are inherently difficult to diagnose clinically, particularly those not fulfilling the classical ABCD criteria (asymmetry, border irregularity, colour variegation, and diameter $>6 \mathrm{~mm}) .{ }^{3}$ In one study, nodular, desmoplastic and ALM subtypes were not only associated 
with rapid and aggressive tumour growth but were also more likely to be clinically atypical. ${ }^{4}$ That is, they were more often amelanotic, symmetrical and elevated, with a regular border. Diagnostic features of these atypical melanomas are less effectively taught, and timely and accurate diagnosis presents a major challenge, particularly in the general practice setting.

Given the difficulties inherent in diagnosis, misdiagnosis of an atypical melanoma should not necessarily be considered to be a breach of a reasonable standard of care, especially given that GPs may see very few of these lesions during their careers. Whether a misdiagnosis constitutes a breach of duty of care is determined by a court on the basis of the admissible evidence, including expert peer professional opinion. In this case, the plaintiff's evidence that there was pigmentation of the lesion at the initial presentation was critical in determining whether a breach of duty had occurred. In the absence of comprehensive clinical notes, it was difficult for the defendant to establish that there was no pigmentation at the initial presentation. All the clinicians involved in this case agreed that if there was pigmentation of the lesion, further investigation would have been warranted, as this might have indicated a diagnosis other than that of a plantar wart. The importance placed by the court on the presence or absence of pigmentation in determining the appropriate response of a reasonably competent practitioner is interesting and emphasised in the appeal judgment. ${ }^{5}$ It was largely based on the expert opinions provided. While the presence or absence of pigmentation may be an appropriate diagnostic clue, it is only one part of the broader clinical picture. The courts may tend to lend it excessive weight. Pigmentation alone should not dispose of the question of breach of duty of care. A more reliable clue to misdiagnosis may well be failed response to treatments that have been tried.

Repeated failed non-definitive treatments were continued by multiple clinicians, which allowed a significant amount of time to pass without the patient being referred to a clinician with peer-recognised specialist qualifications in the diagnosis and management of skin disease. On the evidence available, we consider that it was not the misdiagnosis per se that amounted to a breach of duty of care, but the lack of recognition of uncertainty and a failure to appropriately refer the patient or conduct further investigations once treatment failure became apparent. Definitive biopsy or escalation of care by referral to a specialist may each have averted the breach in this case.

From a clinical perspective, this reinforces the importance of accurate and precise documentation and personal communication with other clinicians. A change in presentation or a pattern of unsuccessful treatment, and hence uncertainty in diagnosis, can thereby be identified and acted on.

\section{Causation and prognosis}

\section{Judicial findings}

The court of first instance held that the evidence was insufficient to prove that an improved prognosis was probable, rather than possible, had the patient's ALM been diagnosed and treated at first presentation. In the absence of proven causation of damage, despite a proven breach of duty of care, the court rejected the claim in negligence.

\section{Commentary}

The relationship between delay in diagnosis and poorer prognosis in progressive neoplastic disease may seem intuitive for many clinicians. The court's decision on this point may therefore seem surprising. Indeed, the Court of Appeal rejected it.

The Breslow thickness of a melanoma at the time of removal is a major predictor of the likelihood of metastasis and therefore of overall prognosis. ${ }^{5}$ In Australia, the 10year survival rate is $98 \%$ for lesions less than $0.76 \mathrm{~mm}$ thick but only $53 \%$ for lesions more than $3 \mathrm{~mm}$ thick; the outcome for people with distant metastasis is extremely poor (5-year survival rate, $<5 \%){ }^{6}$

Despite this, any direct relationship between delay in diagnosis and increased melanoma thickness remains controversial. ${ }^{6-9}$ It is recognised that melanomas vary widely in their rate of progression, particularly according to subtype, with certain subtypes such as nodular melanoma known to have a rapid vertical growth phase. One explanation for the apparent lack of a demonstrated relationship between diagnostic delay and tumour thickness is that tumour thickness at diagnosis may be more strongly related to the growth rate and biological aggressiveness of the tumour, rather than to the measured delay in diagnosis. ${ }^{9}$ Considering melanomas together as a homogeneous group, rather than as subtypes with widespread variability in rates of growth, has also been suggested to be a possible confounding factor for any measured association between thickness and delay in diagnosis. ${ }^{5}$ It therefore becomes difficult to retrospectively draw conclusions to determine the prognostic impact of misdiagnosis.

The court referred to the recent landmark High Court case of Tabet $v$ Gett,${ }^{10}$ where a claim of negligence resulting only in a loss of a chance of a better medical outcome was rejected. Tabet $v$ Gett has authoritatively settled the point that the defendant's negligence must be proven, on the balance of probabilities, to be the cause of the poorer outcome for the patient, compared with the expected outcome had the breach of duty not occurred. A loss of a chance of a better outcome is not of itself sufficient for a claim in negligence to succeed.

In general, in Australia, for there to be factual causation, it is necessary to prove that the harm to the plaintiff would not have occurred without the defendant's breach. In Coote $v$ Dr Kelly, the breach by the defendant was effectively that of misdiagnosing melanoma and delaying targeted treatment. The key question therefore became whether it could be proven that a difference existed between the prognosis for the patient at the time of the first presentation and the prognosis at the time that the melanoma was eventually diagnosed, and whether this difference was caused by the actions of his GP.

Much of the evidence relied on the interpretation by expert witnesses of epidemiological studies used for determining possible prognosis. The purpose of these studies is not to establish likely prognosis or to determine 
retrospective prognosis in any particular case. As a consequence, on the balance of probabilities, the firstinstance court determined it was not proven that metastasis had not already occurred and, at the time of his initial presentation in 2009, this particular patient may have already had a poor prognosis.

The Court of Appeal expressly rejected the proposition that epidemiological studies cannot provide evidence sufficient to prove causation on the balance of probabilities of loss in an individual case. We respectfully agree with the Court of Appeal and strongly caution the medical profession against relying on an assertion (supported at first instance) that epidemiological evidence is incapable of supporting a legal finding of causation in any individual case. It is so capable. "There is nothing in Tabet $v$ Gett... standing in the way of such a conclusion" ${ }^{11}$

Melanomas with rapid vertical growth phases and aggressive histological features may have vertical progression rates $>0.5 \mathrm{~mm}$ in thickness per month ${ }^{4}$ and, in these cases, it is even more likely that delay in diagnosis may lead to a significant decline in prognosis. The importance of accurate and timely diagnosis of such lesions, in order to capitalise on a potentially short window of opportunity for improved prognosis, is a clinical imperative granted additional legal force by the recent Court of Appeal findings.

\section{Conclusion}

A high index of suspicion is always necessary when considering diagnoses that require early intervention to prevent significant harm to the patient. It may be difficult to diagnose atypical presentations of melanoma. By their very nature, such melanomas will have a higher rate of misdiagnosis.

Failure of initial treatment should trigger a recognition of uncertainty, an awareness of possible serious differential diagnoses and an understanding of the potential significance of error, with consequent escalation to specialist diagnostic and clinical care. Such recognition may be evident on the first consultation, or it may take several consultations before a pattern of uncertainty emerges. Consistency in documentation and communication among colleagues is essential where continuity of care is suboptimal. Recognising uncertainty and the need for escalation when recognised is a principle that applies to all aspects of clinical and histopathological practice. Its importance in the context of melanoma diagnosis, where delay may be a critical factor in the patient's ultimate survival, is paramount. Establishing causation as a result of delayed diagnosis is often complicated, but it is possible, and epidemiological evidence may be employed to do it. If the litigation is not settled prior to retrial, the authors propose to publish further analysis of this important case as any judgment may become available.

Competing interests: No relevant disclosures.

Provenance: Not commissioned; externally peer reviewed.

1 Coote v DrKelly [2012] NSWSC 219.

2 Coote v Kelly (2013) NSWCA 357.

3 Friedman RJ, Rigel DS, Kopf AW. Early detection of malignant melanoma: the role of physician examination and self-examination of the skin. CA Cancer J Clin 1985; 35: 130-151.

4 Liu W, Dowling JP, Murray WK, et al. Rate of growth in melanomas, characteristics and associations of rapidly growing melanomas. Arch Dermatol 2006; 142: 1551-1558.

5 Marks R. The changing incidence and mortality of melanoma in Australia. Recent Results Cancer Res 2002; 160: 113-121.

6 Baade PD, English DR, Youl PH, et al. The relationship between melanoma thickness and time to diagnosis in a large population-based study. Arch Dermatol 2006; 142: 1422-1427.

7 Krige JEJ, Isaacs S, Hudson DA, et al. Delay in the diagnosis of cutaneous malignant melanoma. A prospective study in 250 patients. Cancer 1991; 68: 2064-2068.

8 Richard MA, Grob JJ, Avril MF, et al. Delays in diagnosis and melanoma prognosis (II): the role of doctors. Int J Cancer 2000; 89: 280-285.

9 Richard MA, Grob JJ, Avril MF, et al. Melanoma and tumour thickness, challenges of early diagnosis. Arch Dermatol 1999; 135: 269-274.

10 Tabet v Gett [2010] HCA 12.

11 Coote v Kelly (2013) NSWCA 357: 64. 